\title{
Intravitreal methotrexate infusion for proliferative vitreoretinopathy
}

\author{
This article was published in the following Dove Press journal: \\ Clinical Ophthalmology \\ 19 September 2016 \\ Number of times this article has been viewed
}

\author{
Ama Sadaka' \\ Robert A Sisk ${ }^{1-3}$ \\ James M Osher ${ }^{1,3}$ \\ Okan Toygar ${ }^{4}$ \\ Melinda K Duncan ${ }^{5}$ \\ Christopher D Riemann ${ }^{1,3}$ \\ 'Department of Ophthalmology, \\ University of Cincinnati College \\ of Medicine, ${ }^{2}$ Department of \\ Opthalmology, Cincinnati Children's \\ Hospital Medical Center, ${ }^{3}$ Cincinnati \\ Eye Institute, Cincinnati, OH, USA; \\ ${ }^{4}$ Department of Ophthalmology, \\ Bahcesehir University Medical Faculty, \\ Istanbul, Turkey; ${ }^{5}$ Department of \\ Biological Sciences, University of \\ Delaware, Newark, DE, USA
}

Purpose: The purpose of this study was to evaluate intravitreal methotrexate infusion (IMI) during pars plana vitrectomy (PPV) for retinal detachment in patients with high risk for the development of proliferative vitreoretinopathy (PVR).

Methods: Patients presenting with severe recurrent PVR with tractional retinal detachment and/or a history of severe ocular inflammation were treated with IMI. Clinical outcomes were determined from a retrospective medical chart review.

Results: Twenty-nine eyes presenting with either tractional retinal detachment and recurrent PVR $(n=22)$ or a history of severe inflammation associated with high PVR risk $(n=7)$ received IMI during PPV. Best-corrected visual acuity at 6 months was $\geq 20 / 200$ in 19 of 29 eyes (66\%) and remained stable or improved compared with initial presentation in 24 of 29 eyes (83\%). At the last follow-up examination, the retinas of 26 of 29 eyes $(90 \%)$ remained attached after IMI while three eyes required another reattachment procedure. Three additional eyes $(10 \%)$ developed recurrent limited PVR without recurrent RD and were observed. No complications attributable to IMI occurred during a mean follow-up of 27 months.

Conclusion: Eyes at high risk for PVR development due to a history of prior PVR or intraocular inflammation had a low incidence of PVR following IMI at the time of PPV for RD repair. No significant safety issues from IMI were observed in this series.

Keywords: tractional retinal detachment, recurrent retinal detachment, pars plana vitrectomy

\section{Introduction}

Proliferative vitreoretinopathy (PVR) is defined by the presence of peri-retinal fibrocellular membranes the contraction of which can lead to rhegmatogenous retinal detachment (RRD). PVR affects 5\%-10\% of retinal detachment (RD) patients and is present in $75 \%$ of failed RD repairs. PVR seems to be caused by the migration of retinal pigment epithelial (RPE) cells from their normal location to the peri-retina following a break in the retina or breakdown in the blood-retinal barrier. These mislocated RPE cells proliferate and undergo epithelial to mesenchymal transition (EMT) into contractile myofibroblasts that produce the extracellular matrix of the fibrocellular membrane. ${ }^{1}$ The EMT of RPE cells occur coincidently with the activation of glia, immune cells, and astrocytes, which proliferate and migrate on both the sides of the detached retina, increasing the complexity of the cellular organization of the fibrocellular membranes. ${ }^{2}$

Anti-inflammatory drugs such as corticosteroids have been used as adjuvants to retinal reattachment surgery to prevent PVR since preexisting ocular inflammation is a known PVR risk factor, and inflammatory cells are found associated with periretinal membranes. ${ }^{3}$ However, the efficacy of anti-inflammatory treatment in improving retinal reattachment rates, vitreous cell reaction, and PVR are mixed. ${ }^{4}$ Similarly,
Correspondence: Christopher D Riemann 1945 CEl Drive, Cincinnati,

$\mathrm{OH} 45242$, USA

Tel +I 5139845133

Fax +I 5139842390

Email criemann@cincinnatieye.com 
antimetabolites such as 5-fluorouracil have been tested as PVR therapies to prevent the cell proliferation seen in PVR, but no consistent efficacy has been demonstrated. ${ }^{4,5}$

Methotrexate (MTX) is a commonly used antineoplastic agent since it is a potent competitive inhibitor of enzymes requiring folate as a cofactor, including those critical for the nucleotide biosynthesis necessary for the DNA synthesis required for cell proliferation. ${ }^{6}$ Notably, lower concentrations of MTX are effective treatments for chronic inflammatory conditions such as rheumatoid arthritis and psoriasis, likely due to the ability of its metabolite, MTX polyglutamate, to inhibit the enzyme aminoimidazole carboxamide ribonucleotide transformylase, resulting in elevated extracellular concentrations of adenosine, a potent anti-inflammatory agent. ${ }^{7}$ MTX has also been commonly used to treat fibrotic conditions such as keloid, where in addition to its ability to inhibit cell proliferation and inflammation, it may have a direct anti-fibrotic effect as treated fibroblasts make less type I collagen than controls. ${ }^{8}$ Thus, MTX has the potential to block many aspects of PVR simultaneously including aberrant cell proliferation, inflammation, and fibrosis.

In ophthalmology, intraocular MTX has been used to treat numerous inflammatory conditions such as juvenile idiopathic arthritis-associated uveitis, sarcoidosis-related panuveitis, mucous membrane pemphigoid, rheumatoid-associated scleritis and episcleritis, sympathetic ophthalmia, corticosteroid-resistant uveitis ${ }^{9}$ while higher doses are used to treat primary intraocular lymphoma. ${ }^{10}$ Overall, intraocular MTX therapy has an excellent safety record both in animal models and in clinical practice, ${ }^{11,12}$ although corneal epitheliopathy and maculopathy have been reported in patients receiving multiple intraocular MTX injections for lymphoma. ${ }^{13}$

A recent report included $250 \mu \mathrm{g}$ of MTX in the silicon oil tamponade instilled at the end of retinal reattachment surgery in advanced diabetic retinopathy patients without significant adverse effects; however, clinical efficacy in preventing recurrent $\mathrm{RD}$ in diabetic retinopathy was not observed. ${ }^{14}$

In the present study, intravitreal MTX infusion (IMI) is used during pars plana vitrectomy (PPV) in patients at high risk of PVR development and its effects on recurrent RD and final visual outcomes are assessed.

\section{Materials and methods}

This is a consecutive case series pilot study. Our cohort consisted of consecutive patients who presented with RD between 2008 and 2014 and received IMI during surgical repair of RD with PPV. Preoperative history, surgical details, and postoperative course were retrieved from electronic medical records. PVR was graded following the standard retina society classification, $1983 .{ }^{4}$

The Cincinnati Eye Institute Institutional Review Board approval for a retrospective chart review was obtained. All patients signed an informed consent for surgery with off-label use of MTX after a detailed discussion of risks, benefits, alternatives, and unknowns of this intervention. All patient data were collected in strict compliance with the Declaration of Helsinki and the United States Health Insurance Portability and Accountability Act.

\section{Study population}

Patients were evaluated and treated by two surgeons, CDR and RAS, at the Cincinnati Eye Institute (CEI). Patients selected for IMI exhibited severe ocular pathology in which recurrent PVR was already present or they were at very high risk for PVR due to their clinical profile. These patients were often referred to CEI for tertiary care after having multiple failed previous surgeries at other institutions. Patients were considered to have PVR if they had stage $\mathrm{C}$ PVR (Retina Society Classification, 1983) present in one or more quadrants. Isolated epiretinal membranes were not considered as PVR.

The indications for IMI are outlined in Table 1. All subjects who received IMI during PPV performed by CDR and RAS during the inclusion period are included in this series.

\section{Surgical technique}

Forty milligrams of MTX was added to each $500 \mathrm{~mL}$ balanced saline solution infusion bottle used during surgery. This infusion-based dosing approach was selected to assure similar dosing across all patients irrespective of the volume of infusion fluid used during the surgical procedure. This dosing is thought to yield intraocular MTX levels similar to that used in

Table I Indications for PPV and IMI

Recurrent tractional retinal detachment with proliferative vitreoretinopathy $(\mathbf{n}=\mathbf{2 2})$

History of multiple failed retinal detachment repairs $(n=16)$

Perforating globe injuries $(n=3)$

History of proliferative diabetic retinopathy and TRD repair $(n=2)$

Retro-keratoprosthetic membrane and anterior TRD $(n=l)$

Primary vitrectomy surgery with a history of inflammatory complications $(\mathbf{n}=\mathbf{7})$

Primary RRD repair with a history of contralateral PVR $(n=3)$

Primary RRD with concurrent severe inflammatory disease $(n=2)$

Severe ERM with tractional changes and inflammatory disease $(n=2)$

Abbreviations: ERM, epiretinal membrane; IMI, intravitreal methotrexate infusion; PPV, pars plana vitrectomy; RRD, rhegmatogenous retinal detachment; TRD, tractional retinal detachment. 
intraocular lymphoma treatment (400 $\mu \mathrm{g}$ intravitreal injection of MTX into an $\sim 5 \mathrm{~mL}$ volume of a human eye). ${ }^{7,15}$

All surgeries were performed with the Constellation Vitrectomy System (Alcon, Ft Worth, TX, USA). A 25-, 23-, or 20-gauge surgery was performed according to the surgeon's preferences. Scleral depressed and bimanual vitrectomy were performed to visualize the anterior retina and vitreous base. Ocular endoscopy was employed for visualization through media opacity or to visualize anterior structures as needed. Membrane peeling of PVR and endolaser were performed in all eyes. Scleral buckling, relaxing retinectomy, perfluoron injection, silicone oil tamponade, and gas tamponade with SF6 or C3F8 were performed according to the intraocular situation and surgeon preference.

\section{Main outcome measures and statistical analysis}

Preoperative and 6-month postoperative visual acuities, retinal attachment status, and the presence of new PVR with or without recurrent RD were the main outcome measures. Snellen visual acuities were converted to logarithm of the minimum angle of resolution (logMAR) for analysis. Counting fingers (CF), hand motion (HM), and light perception (LP) vision were considered 20/2,000,20/20,000, and 20/200,000, respectively. Student's $t$-test was used for comparison using Microsoft Excel 2011. A $P$-value of $<0.05$ was considered significant.

\section{Results}

\section{Demographics}

Table 2 summarizes the clinical data. Twenty-nine eyes of 28 patients (17 male; 17 right eyes) with a mean age of 56 years (range 10-77 years) received IMI during PPV. Eyes had a median of two PPVs prior to surgery with IMI (range $0-6$ surgeries). PPV was 25-gauge in 22 eyes (76\%), 23 -gauge in five eyes (17\%), and 20-gauge in two eyes (7\%). Ocular endoscopy was employed in two eyes $(7 \%)$. Membrane peeling of PVR was performed in 26 eyes (90\%), and endolaser was performed in all eyes. Epiciliary membranes were removed in two eyes (7\%). Mean follow-up was 27 months (range 9-63 months).

\section{Response to treatment}

Table 1 summarizes the indications for PPV with MTX infusion. No complications were directly attributable to IMI. Anterior chamber inflammation was grade $2+$ or less without fibrin formation in all eyes that received IMI. No patient developed an epithelial defect if the corneal epithelium was not scraped at the time of surgery, and no patient had a persistent epithelial defect. Visual acuity (VA) prior to PPV with IMI ranged from 20/70 to LP. At 6 months, VA ranged from 20/20 to LP. It improved in 21 eyes, remained stable in five eyes, and worsened in three eyes. Of those three eyes, one patient had proliferative diabetic retinopathy that had been treated with panretinal photocoagulation previously (patient no 3), one had a history of sarcoid uveitis and developed hypotony after surgery (patient no 12), and the third patient had no ocular disease prior to the RD (patient no 23). Nineteen of 29 eyes $(66 \%)$ had 20/200 vision or better at 6 months (Figure 1). Paired Student's $t$-test showed statistical significance when comparing preoperative to postoperative VA at 6 months $(P=0.0002)$. VA data showed both statistically significant VA gains. Mean VA was CF (range 20/70 to LP) preoperatively and improved to 20/250 (range 20/20 to LP, $P<0.0002$ ) postoperatively. Ambulatory vision (CF or better) was present in 14 of 29 eyes (48\%) preoperatively and improved to 21 of 29 eyes ( $72 \%$ ) postoperatively. The vision was $20 / 200$ or better in 10 of 29 eyes (34\%) preoperatively and 18 of 29 eyes (62\%) postoperatively. 20/60 or better VA was present in one of 29 eyes $(3 \%)$ preoperatively and ten of 29 eyes (34\%) postoperatively.

All retinas were reattached at the conclusion of surgery. At postoperative month 6, the retinas of 26 of 29 eyes $(90 \%)$ remained attached, but three eyes developed recurrent RD from PVR. Two eyes were treated with laser retinopexy and one eye underwent reoperation with PPV for recurrent RD. Among those eyes, two had a history of open-globe injuries, and one was an aniridic patient with a retro-keratoprosthetic membrane and anterior tractional RD. At the last follow-up, three additional eyes (10\%) developed recurrent PVR without recurrent RD that did not require further intervention. All three of these had a history of open-globe injury. Five eyes had a history of significant inflammation prior to IMI. Only one of those eyes (patient no 12) had recurrent PVR and had decline in vision after surgery (from 20/400 to HM vision). This patient had a history of severe sarcoid uveitis and developed hypotony with choroidals postoperatively.

Three patients had a history of previous contralateral postoperative PVR and recurrent RD after PPV surgery without IMI use. In those three patients, the contralateral eye had the same initial pathology as the eye that received IMI: one had macular hole, the second had RD, and the third had pars planitis and developed PVR after epiretinal membrane peeling. For those patients, the fellow eyes that received IMI did not experience recurrent RD or PVR (Tables 1 and 2). 
Table 2 Summary of patients' clinical data

\begin{tabular}{|c|c|c|c|c|c|c|c|}
\hline $\begin{array}{l}\text { Patient } \\
\text { ID }\end{array}$ & Indication for MTX & Comorbidities & $\begin{array}{l}\text { No of } \\
\text { previous } \\
\text { surgeries }\end{array}$ & $\begin{array}{l}\text { Preoperative } \\
\text { VA }\end{array}$ & $\begin{array}{l}\text { Postoperative } \\
\text { VA at } \\
6 \text { months }\end{array}$ & $\begin{array}{l}\text { Months of } \\
\text { follow-up }\end{array}$ & $\begin{array}{l}\text { Anatomical } \\
\text { outcome }\end{array}$ \\
\hline 1 & Recurrent PVR with RD & $\begin{array}{l}\text { Severe inflammation and } \\
\text { perforating globe injury }\end{array}$ & 6 & $20 / 160$ & $20 / 80$ & 63.25 & Attached with no PVR \\
\hline 2 & Recurrent PVR with RD & PDR & 2 & $20 / 80$ & $20 / 50$ & 37 & Attached with no PVR \\
\hline 3 & Recurrent PVR with RD & PDR & I & $20 / 70$ & $20 / 200$ & 52.25 & Attached with no PVR \\
\hline 4 & Recurrent PVR with RD & & 4 & $20 / 125$ & $20 / 60$ & 23.75 & Attached with no PVR \\
\hline 5 & Recurrent PVR with RD & & 3 & LP & LP & II & Attached with no PVR \\
\hline 6 & Recurrent PVR with RD & & 2 & $\mathrm{CF}$ & $20 / 250$ & 42.5 & Attached with no PVR \\
\hline 7 & Recurrent PVR with RD & $\mathrm{MH}$ and $\mathrm{HST}$ & 3 & $\mathrm{CF}$ & $20 / 400$ & 30.75 & Attached with limited PVR \\
\hline 8 & $\begin{array}{l}\text { Primary RD with } \\
\text { contralateral PVR }\end{array}$ & $\mathrm{MH}$ & 0 & $20 / 70$ & $20 / 40$ & 15.25 & Attached with no PVR \\
\hline 9 & Recurrent PVR with RD & & I & $20 / 160$ & $20 / 160$ & 36 & Attached with no PVR \\
\hline 10 & $\begin{array}{l}\text { Primary RD with severe } \\
\text { inflammation }\end{array}$ & & 0 & CF & $20 / 200$ & 26.75 & Attached with no PVR \\
\hline II & $\begin{array}{l}\text { ERM with severe } \\
\text { inflammation }\end{array}$ & & 2 & $20 / 400$ & $20 / 20$ & 79.5 & Attached with no PVR \\
\hline 12 & $\begin{array}{l}\text { Primary RD with severe } \\
\text { inflammation }\end{array}$ & $\begin{array}{l}\text { Severe sarcoid } \\
\text { uveitis with hypotony } \\
\text { maculopathy } \\
\text { postoperatively }\end{array}$ & 1 & $20 / 400$ & HM & 52.25 & Attached with limited PVR \\
\hline 13 & $\begin{array}{l}\text { Primary RD with } \\
\text { contralateral PVR } \\
\text { following ERM peel }\end{array}$ & ERM, pars planitis & 0 & $20 / 400$ & $20 / 40$ & 11.75 & Attached with no PVR \\
\hline 14 & $\begin{array}{l}\text { Primary RD with } \\
\text { contralateral PVR after } \\
\text { RD repair }\end{array}$ & & 0 & $\mathrm{CF}$ & $20 / 25$ & 10.5 & Attached with no PVR \\
\hline 15 & Recurrent PVR with RD & Perforating globe injury & 4 & HM & $\mathrm{CF}$ & 36.75 & Attached with limited PVR \\
\hline 16 & Recurrent PVR with RD & Perforating globe injury & I & HM & HM & 29 & Attached with limited PVR \\
\hline 17 & Recurrent PVR with RD & & 2 & $\mathrm{CF}$ & $20 / 30$ & 29.75 & Attached with no PVR \\
\hline 18 & Recurrent PVR with RD & & 2 & $\mathrm{HM}$ & $20 / 200$ & 36.25 & Attached with no PVR \\
\hline 19 & $\begin{array}{l}\text { ERM with severe } \\
\text { inflammation }\end{array}$ & & 1 & $20 / 50$ & $20 / 40$ & 23.25 & Attached with no PVR \\
\hline 20 & Recurrent PVR with RD & & I & $20 / 100$ & $20 / 70$ & 13 & Attached with no PVR \\
\hline 21 & Recurrent PVR with RD & Perforating globe injury & I & $\mathrm{HM}$ & $20 / 60$ & 11.25 & Attached with limited PVR \\
\hline 22 & Recurrent PVR with RD & & I & $20 / 100$ & $20 / 60$ & 17.25 & Attached with no PVR \\
\hline 23 & Recurrent PVR with RD & & 2 & $20 / 70$ & $20 / 80$ & 19 & Attached with no PVR \\
\hline 24 & Recurrent PVR with RD & & I & CF & $20 / 100$ & 12 & Attached with no PVR \\
\hline 25 & Recurrent PVR with RD & & 2 & $\mathrm{CF}$ & $20 / 125$ & 9.25 & Attached with no PVR \\
\hline 26 & Recurrent PVR with RD & & I & $\mathrm{CF}$ & CF & 18.25 & Attached with no PVR \\
\hline 27 & Recurrent PVR with RD & $\begin{array}{l}\text { Boston keratoprosthesis } \\
\text { retromembrane }\end{array}$ & I & HM & $\mathrm{CF}$ & 16.5 & Attached with no PVR \\
\hline 28 & Recurrent PVR with RD & & 2 & $\mathrm{CF}$ & $\mathrm{CF}$ & 10.25 & Attached with no PVR \\
\hline 29 & Recurrent PVR with RD & & I & LP & HM & 17.75 & Attached with no PVR \\
\hline
\end{tabular}

Abbreviations: CF, counting fingers; ERM, epiretinal membrane; HM, hand motions; HST, horse shoe tear; LP, light perception; MH, macular hole; MTX, methotrexate; PDR, proliferative diabetic retinopathy; PVR, proliferative vitreoretinopathy; RD, retinal detachment; VA, visual acuity.

\section{Discussion}

PVR remains a persistent problem complicating RRD repair despite many surgical advancements in the management of RD. ${ }^{16}$ Patients are at higher risk of developing PVR if their clinical presentation includes penetrating globe injury, multiple tears, giant tears, a larger RD ( $>2$ quadrants), vitreous hemorrhage, preoperative PVR, preoperative and postoperative choroidal detachment, uveitis, inflammation with anterior chamber and vitreous flare, aphakia, use of vitrectomy, cryoretinopexy, or heavy endolaser application for RD repair, and repeated surgical procedures. ${ }^{2,16}$ All our patients had two or more of these risk factors.

The pathophysiology of PVR is complex and likely requires the cooperation of multiple cell types. Following $\mathrm{RD}$, glial cells are activated and contribute to the formation of membranes in PVR. RPE cells lose their polarity, dedifferentiate, and undergo epithelial to mesenchymal transition, converting to myofibroblasts in response to unregulated growth factor exposure. This phenotypic transformation creates cells that, unlike their RPE progenitors, 


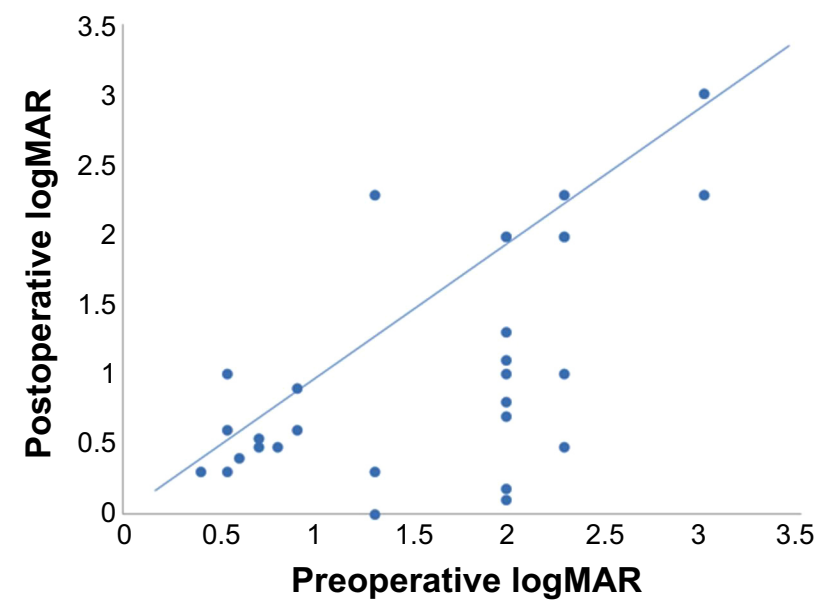

Figure I Distribution of preoperative and 6 months postoperative $\log M A R$ visual acuities before and after IMI $(P=0.0002)$.

Abbreviations: IMI, intravitreal methotrexate infusion; logMAR, logarithm of the minimum angle of resolution.

are proliferative, migratory, contractile, and capable of the robust extracellular matrix secretion necessary to form the fibrocellular membranes diagnostic of PVR. Macrophages are either recruited to the site of RD or formed from other retinal cells in response to molecules secreted by the injured retina. While macrophages can be beneficial for removing cellular debris, they also drive PVR by secreting pro-inflammatory cytokines that convert RPE cells into myofibroblasts. ${ }^{2}$ The role of pro-inflammatory cytokines in PVR likely explains why most of the risk factors for PVR development involve inflammation or wound-healing responses. ${ }^{2,16}$

Surgery remains the standard treatment for PVR, yielding an anatomic success rate of $60 \%-90 \%$ with functional success (ambulatory vision $5 / 200$ or better) in only $40 \%-80 \%$ of patients. ${ }^{2}$ As inappropriate cell proliferation is necessary for PVR, most attempts to pharmacologically arrest PVR have focused either on anti-neoplastic agents such as daunorubicin and 5-fluorouracil, which block the ability of proliferative cells to synthesize new DNA, or to couple those agents with low molecular weight heparin to block fibrin formation, theoretically reducing the concentration of growth factors driving PVR. However, although these agents work well in animal models, the results in human patients are mixed, ${ }^{2}$ which has prevented their widespread acceptance for clinical use. Similarly, attempts to block the inflammatory processes critical for PVR with the administration of steroidal anti-inflammatory agents such as dexamethasone and triamcinolone have shown limited efficacy in improving retinal reattachment rates, VA, or PVR reoccurrence in the clinic. ${ }^{2}$

An ideal PVR therapy needs to be safe for intraocular administration while also simultaneously blocking multiple drivers of PVR pathogenesis. High-dose MTX (400 $\mu \mathrm{g}$ administered intravitreally) has been used for 50 years as an effective therapy for intraocular lymphoma due to its ability to stop cell proliferation via its ability to block the de novo nucleotide synthesis needed for DNA synthesis. However, MTX has potent anti-inflammatory activity at concentrations too low to affect normal cellular proliferation, leading to its clinical use in rheumatologic diseases, psoriasis, and ocular inflammatory diseases such as uveitis and scleritis. Thus, we hypothesized that intravitreal use of MTX would safely block the cellular proliferation associated with PVR shortly after administration and then would continue to block the tissue inflammation that drives PVR even as its tissue concentrations decline with time. As MTX is a small molecule with a molecular weight of $454.44 \mathrm{~g} / \mathrm{mole}$, it achieves stable tissue concentrations early in the course of vitrectomy in animal models. ${ }^{11,17}$ Thus, we chose to administer the MTX as a step dosing in the infusion during the vitrectomy portion of the surgery as opposed to bolus dosing at the end of surgery to achieve uniform dosing and bypass concerns of unpredictable MTX concentrations in the setting of silicone oil or gas tamponades. This process does not limit the volume for tamponades or introduce an aqueous phase around retinal breaks that could limit retinal attachment. MTX's efficacy as an anti-neoplastic agent is usually attributed to its ability to inhibit the nucleotide biosynthesis necessary for the DNA synthesis that is necessary for cell proliferation. Numerous studies have shown that, at higher doses, MTX inhibits cell proliferation of numerous cell types including those involved in PVR such as fibroblasts, ${ }^{8}$ astrocytes,${ }^{18}$ and glia. ${ }^{19}$ Its effect on inflammation is observed at concentrations too low to affect cell proliferation.

Rare side effects such as vitreous hemorrhage, maculopathy, endophthalmitis, and corneal epitheliopathy have been reported after repeated intravitreal treatments with doses greater than that used in the present study. ${ }^{9,15}$ No adverse effects of IMI were identified in our small patient cohort, similar to another study that used a similar dose of intraocular MTX to induce the extended remission of uveitis. ${ }^{12}$ Administering only a single dose of MTX may limit the opportunity for toxicity.

Our low rate of recurrent PVR (20\%), low rate of recurrent RD (10\%), and excellent VA results are compared favorably to other studies that evaluated pharmacological interventions for PVR including the Silicone Oil Study. ${ }^{20-23}$ In our series, at 6 months, $83 \%$ of patients had stable or improved VA, and 66\% had VA $\geq 20 / 200$ (Table 3). Perhaps the most intriguing finding in our study was that three patients had a history of contralateral PVR formation causing recurrent $\mathrm{RD}$ prior to presenting with disease in the index eye. In each of these patients, the presenting RD duration and 
Table 3 Main study outcomes

Improved or stable visual acuity at 6 months compared to initial presentation

Visual acuity $\geq 20 / 200$

$24 / 29(83 \%)$

Recurrent PVR without retinal detachment

$19 / 29(66 \%)$

$3 / 29(10 \%)$

Reoperation for recurrent retinal detachment

Abbreviation: PVR, proliferative vitreoretinopathy.

geometry, the specific surgical repair, and the operating surgeon were symmetric in both the eyes. However, the eye that received IMI did not develop ERM, PVR, or recurrent RD. We hypothesize that IMI might be an effective adjuvant to reduce PVR incidence even among eyes without a history of PVR. While a recent report found that addition of MTX to the silicone oil tamponade after retina reattachment did not prevent recurrent detachment in diabetic retinopathy, ${ }^{14}$ the MTX concentrations used were lower than this study and the method of MTX application may limit the access of the drug to the retina. Also, the ability of MTX to induce remission in uveitis suggests that the drug could be particularly efficacious in PVR associated with ocular inflammation. As $>50 \%$ of patients with RD from PVR in one eye develop sight-threatening pathology in the fellow eye, ${ }^{24}$ we intend to investigate the ability of IMI to prevent recurrent PVR with a prospective pilot study in the future.

Our data show promising findings in a small, uncontrolled, retrospective pilot series of selected patients with complex recurrent $\mathrm{RD}$ with PVR, and patients with a history of severe inflammatory complications deemed at high risk for PVR. Although the strength of the conclusions that can be drawn from this study are severely limited due to its small sample size, biased patient selection, lack of control group, and reliance on retrospective chart review, we achieved a remarkable single incisional surgery retinal reattachment success rate of 97\% in this consecutive case series of severely diseased eyes. Another limitation of this study is that our patient cohort is very heterogeneous as outlined in Table 1; however, we felt that it would be important to include all data given the similar outcomes and the nature of pilot studies. Due to the small number of patients in this series and the heterogeneous nature of our patient cohort, there is insufficient statistical power for any detailed analysis of surgical trauma (number of laser spots, number of instrument exchanges, and surgical duration preceding the MTX infusion cases or during the MTX infusion cases) to be meaningful. The surgeries were operated with similar surgical technique, and no one surgery or group of surgeries were strikingly different in terms of surgical trauma than any other.
Despite the existence of these limitations, some important conclusions can be drawn from our data. 1) IMI as an adjuvant to PPV for RD repair seems to be safe. This is expected given its longstanding safety profile in treating other ocular diseases. ${ }^{10}$ 2) VA results at 6 months were excellent considering the severity of ocular disease and were not inferior to other reports in the literature. ${ }^{2,16} 3$ ) The three eyes with severe contralateral PVR that did not develop PVR after IMI highlights further that MTX may be efficacious in PVR prevention. 4) Eyes with significant preoperative inflammation did remarkably well following IMI. As inflammation is a known risk factor for $\mathrm{PVR},{ }^{2}$ it is believed that the ability of MTX to control cell proliferation at the concentrations administered ${ }^{10}$ and its efficacy in controlling inflammation at lower concentrations ${ }^{7,9}$ make IMI a plausible adjuvant therapy for PVR prevention in selected severely diseased eyes with RRD.

PVR remains a challenging complication following RD repair surgery. Overall, the favorable outcomes for this series of high-risk eyes treated for RD with PPV and IMI justify a more rigorous, controlled, prospective study of IMI efficacy. Furthermore, given the low risk of intravitreal MTX administration observed both in this study and in the literature for alternate indications, ${ }^{9,10,15}$ off-label IMI may be considered during RD repair surgery in scenarios where patients are considered at very high risk of developing PVR, such as a history of severe contralateral PVR or a known history of posterior uveitis.

\section{Acknowledgments}

This work was supported in part by an Unrestricted Grant from Research to Prevent Blindness, Inc., New York, NY, to the Department of Ophthalmology, University of Cincinnati, and MKD was supported by National Eye Institute Grant EY015279.

\section{Disclosure}

The authors report no conflicts of interest in this work.

\section{References}

1. Claes C, Lafeta AP. Proliferative vitreoretinopathy. Dev Ophthalmol. 2014;54:188-195.

2. Pastor JC, Rojas J, Pastor-Idoate S, Di Lauro S, Gonzalez-Buendia L, Delgado-Tirado S. Proliferative vitreoretinopathy: a new concept of disease pathogenesis and practical consequences. Prog Retin Eye Res. 2016; 51:125-155.

3. Tosi GM, Marigliani D, Romeo N, Toti P. Disease pathways in proliferative vitreoretinopathy: an ongoing challenge. J Cell Physiol. 2014; 229(11):1577-1583.

4. Sadaka A, Giuliari GP. Proliferative vitreoretinopathy: current and emerging treatments. Clin Ophthalmol. 2012;6:1325-1333. 
5. Sundaram V, Barsam A, Virgili G. Intravitreal low molecular weight heparin and 5-fluorouracil for the prevention of proliferative vitreoretinopathy following retinal reattachment surgery. Cochrane Database Syst Rev. 2013;31(1):CD006421.

6. Sirotnak FM, Moccio DM. Pharmacokinetic basis for differences in methotrexate sensitivity of normal proliferative tissues in the mouse. Cancer Res. 1980;40(4):1230-1234.

7. Chan ES, Cronstein BN. Methotrexate - how does it really work? Nat Rev Rheumatol. 2010;6(3):175-178.

8. Nabai L, Kilani RT, Aminuddin F, Li Y, Ghahary A. Methotrexate modulates the expression of MMP-1 and type 1 collagen in dermal fibroblast. Mol Cell Biochem. 2015;409(1-2):213-224.

9. Gangaputra S, Newcomb CW, Liesegang TL, et al. Methotrexate for ocular inflammatory diseases. Ophthalmology. 2009;116(11):2188-2198. e2181.

10. Frenkel S, Hendler K, Siegal T, Shalom E, Pe'er J. Intravitreal methotrexate for treating vitreoretinal lymphoma: 10 years of experience. Br J Ophthalmol. 2008;92(3):383-388.

11. Manna S, Banerjee RK, Augsburger JJ, Al-Rjoub MF, Donnell A, Correa ZM. Biodegradable chitosan and polylactic acid-based intraocular micro-implant for sustained release of methotrexate into vitreous: analysis of pharmacokinetics and toxicity in rabbit eyes. Graefes Arch Clin Exp Ophthalmol. 2015;253(8):1297-1305.

12. Taylor SR, Banker A, Schlaen A, et al. Intraocular methotrexate can induce extended remission in some patients in noninfectious uveitis. Retina. 2013;33(10):2149-2154.

13. Reichstein D. Primary vitreoretinal lymphoma: an update on pathogenesis, diagnosis and treatment. Curr Opin Ophthalmol. 2016; 27(3):177-184.

14. Ghasemi Falavarjani K, Modarres M, Hadavandkhani A, Karimi Moghaddam A. Intra-silicone oil injection of methotrexate at the end of vitrectomy for advanced proliferative diabetic retinopathy. Eye (Lond). 2015;29(9):1199-1203.

15. Hardwig PW, Pulido JS, Erie JC, Baratz KH, Buettner H. Intraocular methotrexate in ocular diseases other than primary central nervous system lymphoma. Am J Ophthalmol. 2006;142(5):883-885.
16. Pennock S, Haddock LJ, Eliott D, Mukai S, Kazlauskas A. Is neutralizing vitreal growth factors a viable strategy to prevent proliferative vitreoretinopathy? Prog Retin Eye Res. 2014;40:16-34.

17. Ozkan EB, Ozcan AA, Sekeroglu HT, Kuyucu Y, Ozgun H, Polat S. Intravitreal injection of methotrexate in an experimental rabbit model: determination of ultrastructural changes. Indian J Ophthalmol. 2013; 61(7):329-333.

18. Gregorios JB, Soucy D. Effects of methotrexate on astrocytes in primary culture: light and electron microscopic studies. Brain Res. 1990; 516(1):20-30.

19. Serrano EE, Schimke RT. Flow cytometric analysis of mammalian glial cultures treated with methotrexate. Glia. 1990;3(6):539-549.

20. Abrams GW, Azen SP, McCuen BW 2nd, Flynn HW Jr, Lai MY, Ryan SJ. Vitrectomy with silicone oil or long-acting gas in eyes with severe proliferative vitreoretinopathy: results of additional and longterm follow-up. Silicone Study report 11. Arch Ophthalmol. 1997; 115(3):335-344

21. Asaria $\mathrm{RH}$, Kon $\mathrm{CH}$, Bunce $\mathrm{C}$, et al. Adjuvant 5-fluorouracil and heparin prevents proliferative vitreoretinopathy: results from a randomized, double-blind, controlled clinical trial. Ophthalmology. 2001; 108(7):1179-1183.

22. Wiedemann P, Hilgers RD, Bauer P, Heimann K. Adjunctive daunorubicin in the treatment of proliferative vitreoretinopathy: results of a multicenter clinical trial. Daunomycin Study Group. Am JOphthalmol. 1998;126(4):550-559.

23. Kumar A, Nainiwal S, Choudhary I, Tewari HK, Verma LK. Role of daunorubicin in inhibiting proliferative vitreoretinopathy after retinal detachment surgery. Clin Experiment Ophthalmol. 2002;30(5): $348-351$.

24. Schwartz SD, Kreiger AE. Proliferative vitreoretinopathy: a natural history of the fellow eye. Ophthalmology. 1998;105(5):785-788.
Clinical Ophthalmology

\section{Publish your work in this journal}

Clinical Ophthalmology is an international, peer-reviewed journal covering all subspecialties within ophthalmology. Key topics include: Optometry; Visual science; Pharmacology and drug therapy in eye diseases; Basic Sciences; Primary and Secondary eye care; Patient Safety and Quality of Care Improvements. This journal is indexed on Submit your manuscript here: http://www.dovepress.com/clinical-ophthalmology-journal

\section{Dovepress}

PubMed Central and CAS, and is the official journal of The Society of Clinical Ophthalmology (SCO). The manuscript management system is completely online and includes a very quick and fair peer-review system, which is all easy to use. Visit http://www.dovepress.com/ testimonials.php to read real quotes from published authors. 